\title{
Intermittent Fasting: Can It Reduce Insulin Resistance, Improve Insulin Sensitivity, Prevent Diabetes and Metabolic Health Problems
}

\author{
Youngwanichsetha $\mathbf{S}^{*}$ \\ Faculty of Nursing, Prince of Songkla University, Thailand
}

*Correspondence: Sununta Youngwanichsetha, Faculty of Nursing, Prince of Songkla University, Thailand

Received on 09 July 2021; Accepted on 20 July 2021; Published on 15 September 2021

Copyright $(92021$ Youngwanichsetha S. This is an open access article and is distributed under the Creative Commons Attribution License, which permits unrestricted use, distribution, and reproduction in any medium, provided the original work is properly cited.

\section{Editorial}

Diabetes and metabolic health problems are increasing among the population worldwide because of genetic risk factor, unhealthy dietary pattern, and lack of adequate physical activities and exercise. Unhealthy dietary patterns, overconsumption of foods containing sugar, high fructose corn syrup, trans fat, animal fat and processed foods result in overweight, obesity, insulin resistance, and its complications. Reducing insulin resistance and improving insulin sensitivity would be the appropriate intervention to prevent diabetes and metabolic health problems.

Intermittent fasting (IF) is currently interested in modification of dietary patterns in order to reduce insulin resistance and improve insulin sensitivity. Prior studies and lived experiences on IF suggested many health benefits, particularly on improving mitochondrial dysfunction, reducing acumination of advanced glycation end products, and free radicals.

IF is based on religious practices in Buddhism, Islam, Christianity, and others. IF is designed to fast for a longer period than eating time, for example eating time is $8 \mathrm{~h}$, and the fasting period is $16 \mathrm{~h}$. Individuals who practice IF can plan their mealtime, i.e., breakfast during 8.00-10.00 (8-10 am) and dinner during 16.00-18.00 (4-6 pm). There are many methods to do IF, such as the 16/8 method, 5:2 diet, eat-stop-eat pattern, etc. What is the best effective method to do IF should be verified?

On the other hand, types of foods, glycemic index or glycemic load, and portion size should be considered to integrate with the timing of IF practice. Foods containing complex carbohydrates, brown rice, legumes are recommended rather than refined carbohydrates, sugar, fructose, and sweetened drinks. Trans fat, animal fat, and processed foods should be promoted to avoid.

In conclusion, research evidence needs to support the best effective method to do IF and its impacts on prevention and management of diabetes and metabolic health problems.

\section{References}

1. Aman R, Ismail-Beigi F. Intermittent fasting and 'metabolic switch': Effects on metabolic syndrome, prediabetes and type 2 diabetes. Diabetes Obes Metab. 2020;22(9):1496-510.

2. Basaraba S. What is intermittent fasting. 2021. 
3. Dong TA, Sandesara PB, Dhindsa DS, et al. Intermittent fasting: A heart healthy dietary pattern? Am J Med. 2020;133(8):901-907.

4. Grajower MM, Horne BD. Clinical management of intermittent fasting in patients with diabetes mellitus. Nutrients. 2019;11(4):873.

5. Gunnars K. Intermittent fasting 101-The ultimate beginner's guide. 2020.

6. Gunnars K. 6 popular ways to do intermittent fasting. 2021.

7. Khedkar PH. Intermittent fasting: The new lifestyle? Acta Physiol (Oxf). 2020;229(4): e13518.

8. Maideen NMP, Jumale A, Alatrash JIH, et al. Health benefits of Islamic intermittent fasting. J Fasting Health. 2017;5(4):162-71. 